\title{
Methods and Sensor Applications to Analyze Fluid-Structure-Acoustic Problems
}

\author{
Stefan Müller ${ }^{1}$, Stefan Becker ${ }^{1}$ \\ ${ }^{1}$ Institute of Process Machinery and Systems Engineering (iPAT), \\ Fluid System Dynamics and Aeroacoustics, University Erlangen-Nuremberg, \\ Cauerstr. 4, 91058 Erlangen, Germany \\ sb@ipat.uni-erlangen.de
}

\begin{abstract}
:
As a result of the flow around a passenger car, different flat components are excited and, finally, sound is radiated into the passenger cabin. The physical mechanisms which cause this radiation of sound were investigated by using a simplified car model. For the analysis of the exciting wall-pressure fluctuations, a line-array of pressure sensors was used. The structural vibration of the side window was detected by means of a laser-scanning vibrometer. Finally, microphone measurements were performed in the interior. Due to the application of complex analysis techniques to the acquired measurement data, a description of the fluid-structure-acoustic coupling at a passenger car can be given.
\end{abstract}

Key words: vehicle acoustics, wall-pressure, structural vibration, sound radiation, and wavenumber decomposition

\section{Introduction}

The flow-induced excitation of the side windows, in the wake of the a-pillar and the side mirrors, has a significant effect on the interior acoustics for driving speeds above $130 \mathrm{~km} / \mathrm{h}$.

In the present work, the fluid-structure-acoustic coupling was studied in detail. For this purpose, a test case was developed which represents a simplified model of an automobile.

The wall-pressure at the surface of the side window, which can vary in terms of time and space, consists of two different components. The vortex structures in the turbulent, incompressible flow field transported with convection velocity result in hydrodynamic wallpressure fluctuations. Moreover, sound is induced in the flow field which propagates with sound velocity. Corresponding to the disparity of the scales, the sound pressure is masked by the hydrodynamic pressure fluctuations. The identification and quantification of these pressure components require the use of an array of pressure sensors whereby the filtering of the pressure fluctuations according to the associated wavenumbers is possible [1]. Besides, by means of measurements with a line-array in differently overflown areas of the side window it was possible to investigate the Corcos-model [2] with regard to its qualification to model hydrodynamic wall-pressure fluctuations. In the frequency ranges to which the Corcos-model is applicable the model parameters were adapted to the existing flow field.

Due to the similarity of the wavenumbers of the sound pressure and the structural modes, the side window of the model is excited very efficiently by the sound field. Below the critical coincidence frequency of the side window, the non-resonant excitation of structural modes to noise radiation is relevant [3]. Accordingly, the wavenumbers of the structural movement detected by a laser-scanning vibrometer were investigated.

Especially, the supplementary analysis of the sound radiation to the interior for varying flow velocities allows a conclusive explanation for different frequency ranges of the sound pressure spectra.

\section{Model Setup}

The outer geometry of the car model is identical with the SAE Type 4 body (see Fig. 1) [4]. The whole model is acoustically insulated in such a way that noise radiation to the interior is limited to the side window. The streamwise and spanwise extension of the glass plate is illustrated in Fig. 1, corresponding approximately to real side windows. Sheet 
glass with a thickness of $h_{g}=3 \mathrm{~mm}$ was selected. The glass plate is fixed to a frame by means of silicone at each edge over a length of $30 \mathrm{~mm}$. As frame material, a wooden sandwich element with two heavy layers was used. For the experimental investigations, there was no mechanical connection between the side window element and the complemental frame of the car model. The window element lay swimming in a bed of sealing material, and gaps between the surface of the vehicle model and the surface of the window element were sealed and closed with tape. This mounting limits the noise radiation to the interior to the free surface of the side window and prevents transmission of structure-borne sound via the frame.

According to regions with better absorbing characteristics in passenger cars, absorbers were attached to the walls and to the roof interior. In addition, a carpet covered the bottom interior. These absorbers are indispensable to obtain a reverberation time comparable to that in real cars. For the wind tunnel experiments, the model was not mounted directly to the balance of the wind tunnel; rather, there was a damper between the vehicle body and the cylindrical stilts.

In order to induce excitation of the side window, a simplified side mirror was mounted. To induce periodic vortex shedding from the model of the side mirror, a model with square cross-sectional area was attached to the car model. The mirror model with an edge length of $D=80 \mathrm{~mm}$ had a ratio between height and edge length of $H / D=$ 2.5 which is similar to that for real side mirrors.

A free-stream velocity of $U_{\infty}=80,100,120,150$ and $180 \mathrm{~km} / \mathrm{h}$ was applied.

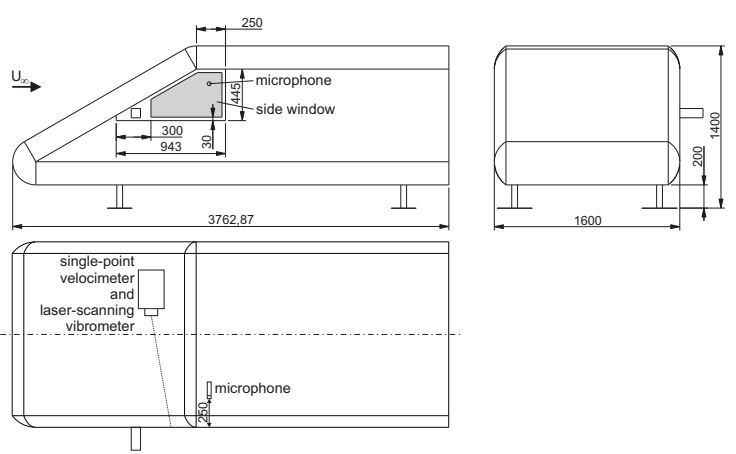

Fig. 1. Schematic drawing of the setup (units in $m m)$.

\section{Experimental Method}

The measurements were performed under semi free field conditions in the acoustic wind tunnel of BMW AG, Munich [4].
To investigate the correlation of different pressure fluctuations at the surface of the side window, correlation measurements of 39 piezoresistive pressure transducers (Kulite XCS-093-1PSID) were carried out. The pressure transducers had a diameter of 2.42 $\mathrm{mm}$ and were inserted into drill holes in an aluminium adaptor, resulting in flush surfaces between the pressure transducers and the adaptor. By substitution of an aluminium side window with cut-outs for the glass side window, the line-array could be placed in different flow regions according to an oil-flow visualization (see Fig. 2).

Phase-resolved laser-scanning vibrometer measurements were carried out to identify the vibration modes of the side window. For this purpose, a Polytec single-point laser-Doppler velocimeter (OFV-505) was used together with a Polytec scanning vibrometer (PSV-400) to detect the vibration velocity of the side window at 1624 uniformly distributed measuring positions.

A 1/2-inch free field condenser microphone (B\&K 4189) was employed for the sound measurements at the position of the driver's ear facing the side window at a distance of $250 \mathrm{~mm}$ (see Fig. 1).

For the microphone measurements, a standard measurement time of $60 \mathrm{~s}$ was used at a sample rate of $96 \mathrm{kHz}$ and, for the correlation measurements, a measurement time of $180 \mathrm{~s}$ was used at a sample rate of $44.1 \mathrm{kHz}$. Simultaneous recording of the different signals was guaranteed by the National Instruments data acquisition system (PXle-1082 with PXle4496 and PXle-4331).

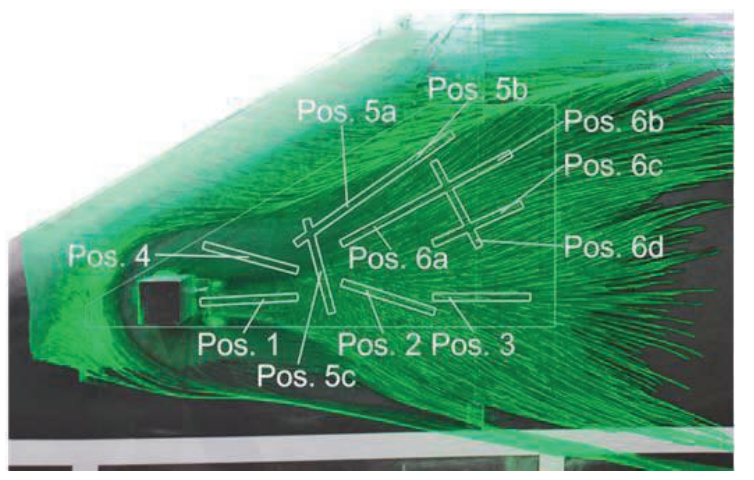

Fig. 2. Oil-flow visualization and positions of the line-array for the acquisition of the wall-pressure fluctuations.

\section{Results}

Unless noted otherwise in the following sections, the results correspond to investigations at a free-stream velocity of $U_{\infty}=$ $150 \mathrm{~km} / \mathrm{h}$. 
Hydrodynamic Wall-Pressure

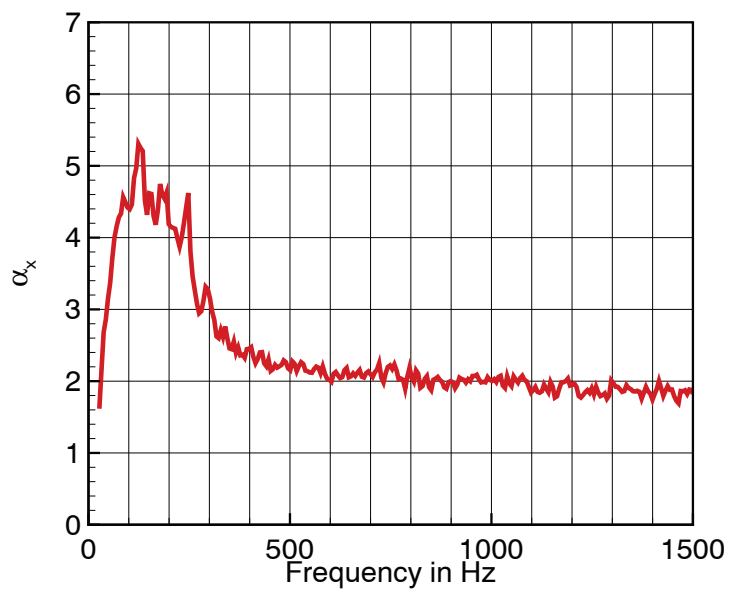

Fig. 3. Spectrum of the Corcos coefficient $\alpha_{x}$ resulting from the array measurement at Pos. 3 (see Fig. 2).

The turbulent flow field exciting the side window of a passenger car is very complex. Therefore, for an efficient simulation of the interior noise the hydrodynamic wall-pressure fluctuations are often modeled. Due to its simplicity and the fact that only two coefficients, $\alpha_{x}$ and $\alpha_{y}$, have to be determined, the Corcos-model [2] is most often applied. This model describes the cross power spectral density (CPSD) by means of the coherence decay of the pressure fluctuations at two positions as the result of the separation of these points at a surface

$$
\begin{aligned}
& G_{x y}(x, y, f)=G_{x x}(f) \cdot e^{-2 \pi \frac{1}{\alpha_{x}} \frac{f}{U_{c}}|x|} . \\
& e^{-2 \pi \frac{1}{\alpha_{y}} \frac{f}{U_{c}}|y|} \cdot e^{j 2 \pi \frac{f}{U_{c}} x} .
\end{aligned}
$$

The coherence, which corresponds to the second and third factor on the right hand side of eq. (1), between the fluctuations at the sensor at a chosen reference position and the subsequent sensor positions can be calculated from the correlation measurements with the line-array. The coherence decay at a discrete frequency gives the value of the corresponding Corcos-coefficient. Figure 3 shows the spectrum of the Corcos-coefficient of the streamwise direction which was determined from the data of the array measurement at Pos. 3 (see Fig. 2). Above $500 \mathrm{~Hz}$, a constant value of 2 can be found for the Corcos-coefficient. This proves that the Corcos-model is applicable for the modelling of the wall-pressure fluctuations induced by the turbulent flow field. Below $500 \mathrm{~Hz}$, large-scale turbulent structures in the wake of the mirror model result in larger coherence lengths which cannot be described by the Corcos model. Taking all array-positions into account, the threshold of $500 \mathrm{~Hz}$ for the application of the Corcos-model has to be raised to $1 \mathrm{kHz}$.

\section{Sound Pressure}

Sound is induced by the fluctuations in the turbulent flow field which propagates with sound velocity. Differently, the vortices are transported with convection velocity. The wavenumber is the ratio between the frequency and the propagation velocity of the sound wave and the turbulent structures, respectively. For the separation and identification of the pressure components, this fact can be used. On the basis of the data of the wall-pressure measurements by means of the line-array, the CPSD of the pressure fluctuations at a reference position and the remaining positions can be calculated. The spatial Fourier-transform of these spectra leads to the wavenumberfrequency spectra [1]

$\Phi\left(k_{x}, f\right)=\int_{-\infty}^{\infty} G_{x y}(x, f) \cdot e^{-j 2 \pi k_{x} x} d x$

In Figure 4, the wavenumber-frequency spectrum of the array measurement at Pos. 3 (see Fig. 2) is depicted. The acoustic part of the wall-pressure fluctuations, with lower wavenumbers, can be separated from the hydrodynamic part at higher wavenumbers. Furthermore, by integration over the related regions the spectra of the different fluctuations can be calculated approximately.

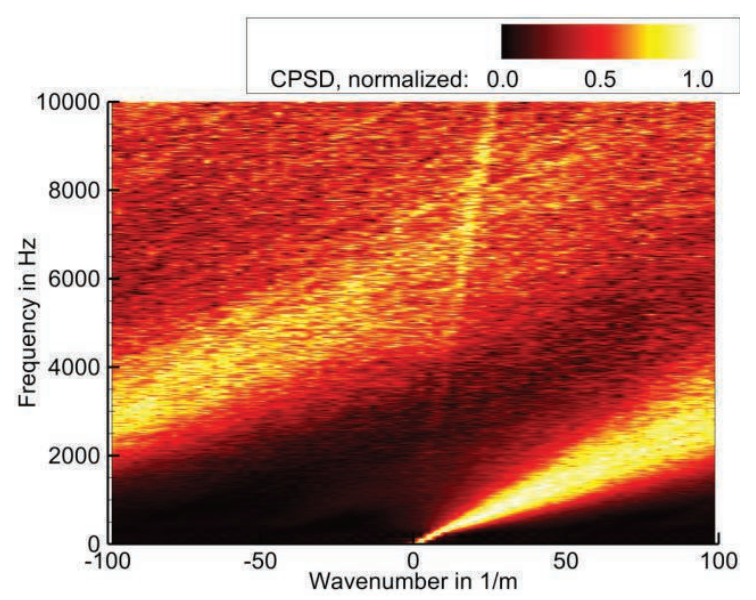

Fig. 4. Wavenumber-frequency spectrum of the CPSD resulting from the array measurement at Pos. 3 (see Fig. 2).

\section{Structural excitation}

Also the spatial Fourier-transform of the structural modes can be calculated which allows for the comparison of the wavenumbers 
of the structural vibration and the exciting pressure fluctuations (see Fig. 5)

$$
S_{m n}\left(k_{x}, k_{y}\right)=\iint_{A} \psi_{m n}(x, y) \cdot e^{-j 2 \pi\left(k_{x} x+k_{y} y\right)} d x d y .
$$

The example for the structural mode with an eigenfrequency of approx. $200 \mathrm{~Hz}$ in Figure 5 shows that the wavenumbers of the structure are enclosed by a circle corresponding to sound pressure fluctuations with a frequency of $2 \mathrm{kHz}$. This means that a non-resonant excitation of this mode by sound pressure is theoretically possible [3]. With regard to the identified sound pressure at the surface of the side window (see Fig. 4), the non-resonant excitation of structural modes of the side window has to be taken into account.

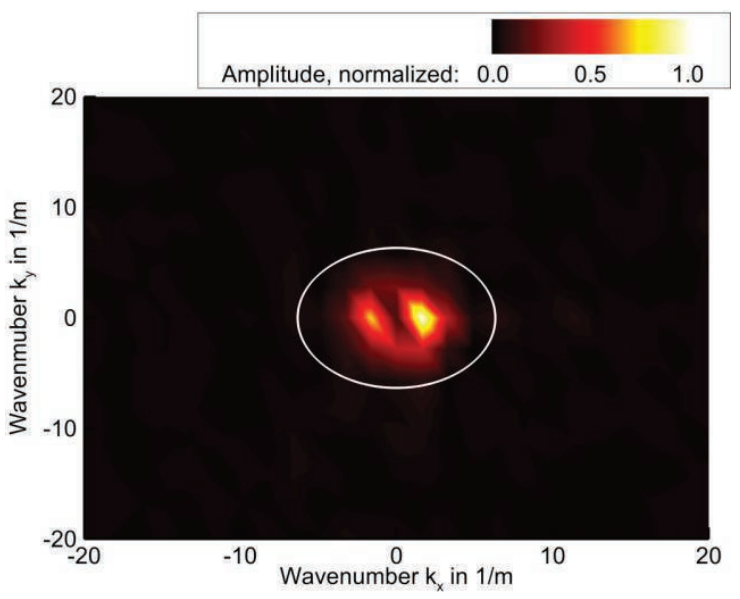

Fig. 5. Spatial Fourier-transform of the structural mode with an eigenfrequency of approx. $200 \mathrm{~Hz}$ (the white circle corresponds to the wavenumber of the sound pressure fluctuations at a frequency of $2 \mathrm{kHz}$ ).

\section{Sound Radiation}

The amplitudes of the spectra of the sound pressure rise with raising free-stream velocity. Scaling of these spectra according to

$$
L_{p, s c a l}=L_{p}-10 \cdot n \cdot \log _{10}\left(\frac{U_{\infty}}{U_{r e f}}\right)
$$

with $n=8$ and $U_{\text {ref }}=150 \mathrm{~km} / \mathrm{h}$ allows for the identification of sound induced by the turbulent flow field [5]. The spectra in Figure 6 coincide to a single curve with the exception of the frequency range around the coincidence frequency of $f_{c} \approx 4 \mathrm{kHz}$. This allows for the conclusion that sound is induced in the turbulent wake flow of a-pillar and side mirror, the sound pressure excites bending waves of the side window and, finally, sound is radiated into the passenger cabin. Considering the results discussed in the previous sections, the non-resonant excitation of structural modes can be given as explanation for the sound radiation below $4 \mathrm{kHz}$. Around the coincidence frequency, the transition to the resonant excitation of modes is observable.

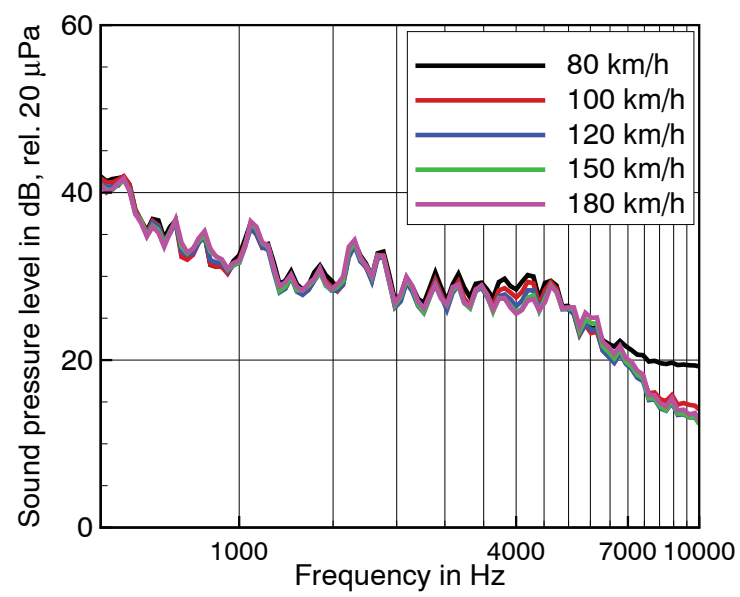

Fig. 6. Scaled spectra of the sound pressure level at the position of the driver's ear (see Fig. 1).

\section{Conclusion}

This work presents a straightforward analysis of the fluid-structure-acoustic coupling at a simplified car model employing different analysis and measurement techniques. The acquisition of the wall-pressure fluctuations by means of a line-array consisting of 39 pressure transducers enabled the proof of the applicability of the Corcos-model to reproduce the hydrodynamic wall-pressure fluctuations for frequencies above $1 \mathrm{kHz}$. In addition, the wavenumber transform of the CPSD of these pressure fluctuations disclosed the presence of sound pressure at the surface of the side window. This sound pressure leads to the excitation of resonant and non-resonant structural modes whereas the requirements for the latter could be confirmed by a transform of the modes to the wavenumber domain.

\section{Acknowledgment}

Financial support by the Bavarian Research Foundation (BFS) is gratefully acknowledged.

\section{References}

[1] B. Arguillat, D. Ricot, C. Bailly, G. Robert, Measured wavenumber: Frequency spectrum associated with acoustic and aerodynamic wall pressure fluctuations, J. Acoust. Soc. Am. 128(4) (2010); doi: 10.1121/1.3478780

[2] G. M. Corcos, Resolution of pressure in turbulence, J. Acoust. Soc. Am. 35(2) (1963); doi: $10.1121 / 1.1918431$ 
[3] F. A. Van Herpe, G. Lobel, P. Lafon, Estimation of automotive wind noise by coupling direct noise computation to statistical energy analysis, 19th

AIAA/CEAS Aeroacoustics Conference (May 2729, 2013, Berlin, Germany), AIAA 2013-2227; doi: 10.2514/6.2013-2227

[4] M. Islam, F. Decker, M. Hartmann, A. Jäger, T. Lemke, J. Ocker, V. Schwarz, F. Ullrich, A.

Schröder, A. Heider, Investigations of sunroof buffeting in an idealised generic vehicle model Part I: Experimental results, 14th AIAA/CEAS Aeroacoustics Conference (May 5-7, 2008, Vancouver, British Columbia Canada), AIAA 2008-2900; doi: 10.2514/6.2008-2900

[5] M. J. Lighthill, On sound generated aerodynamically. I. General theory, Proc. Roy. Soc. A 211(1107) (1952); doi: 10.1098/rspa.1952.0060 